\title{
Erratum: "The Brightest Galaxies in the Dark Ages: Galaxies' Dust Continuum Emission during the Reionization Era" (2018, ApJ, 862, 77)
}

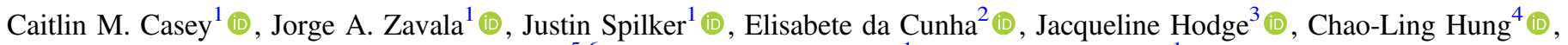
Johannes Staguhn ${ }^{5,6}$ (D) Steven L. Finkelstein ${ }^{1}$ (i) , and Patrick Drew ${ }^{1}$ (1)

${ }^{1}$ Department of Astronomy, The University of Texas at Austin, 2515 Speedway Blvd Stop C1400, Austin, TX 78712, USA; cmcasey@utexas.edu

${ }^{2}$ Research School of Astronomy and Astrophysics, The Australian National University, Canberra ACT 2611, Australia

${ }^{3}$ Leiden Observatory, Niels Bohrweg 2, 2333 CA Leiden, The Netherlands

${ }^{4}$ Department of Physics, Manhattan College, 4513 Manhattan College Pkwy, Bronx, NY 10471, USA

5 NASA Goddard Space Flight Center, Code 665, Greenbelt, MD 20771, USA

${ }^{6}$ Bloomberg Center for Physics and Astronomy, Johns Hopkins University 3400 N. Charles Street, Baltimore, MD 21218, USA Received 2020 February 20; published 2020 March 30

Equations (8) and (9) of the text (also in Table 2) of the published article have a typographical error, where a positive sign was mistakenly printed as a negative sign. This did not impact the analysis of the paper as it was correctly implemented in the authors' code. The correct form of Equation (8), the evolution of $L_{\star}$ with $x$, is

$$
\log _{10} L_{\star}(x)=\frac{\left(\gamma_{2}-\gamma_{1}\right) x_{w}}{2 \pi}\left[\ln \left(\cosh \left(\pi \frac{x-x_{t}}{x_{w}}\right)\right)-\ln \left(\cosh \left(-\pi \frac{x_{t}}{x_{w}}\right)\right)\right]+\frac{\left(\gamma_{2}+\gamma_{1}\right)}{2} x+\log _{10}\left(L_{0}\right) .
$$

Similarly, the correct form of Equation (9), the evolution of $\Phi_{\star}$ with $x$ is

$$
\log _{10} \Phi_{\star}(x)=\frac{\left(\psi_{2}-\psi_{1}\right) x_{w}}{2 \pi}\left[\ln \left(\cosh \left(\pi \frac{x-x_{t}}{x_{w}}\right)\right)-\ln \left(\cosh \left(-\pi \frac{x_{t}}{x_{w}}\right)\right)\right]+\frac{\left(\psi_{2}+\psi_{1}\right)}{2} x+\log _{10}\left(\Phi_{0}\right) .
$$

We thank Chen-Fatt Lim and Arianna Long for bringing this error to our attention.

\section{ORCID iDs}

Caitlin M. Casey (i) https://orcid.org/0000-0002-0930-6466 Jorge A. Zavala (ํ) https://orcid.org/0000-0002-7051-1100 Justin Spilker (1) https://orcid.org/0000-0003-3256-5615 Elisabete da Cunha (i) https://orcid.org/0000-0001-9759-4797 Jacqueline Hodge (i) https://orcid.org/0000-0001-6586-8845
Chao-Ling Hung (i) https://orcid.org/0000-0001-8025-8850 Johannes Staguhn (ํ) https://orcid.org/0000-0002-8437-0433 Steven L. Finkelstein (1) https://orcid.org/0000-00018519-1130

Patrick Drew iㅏ https://orcid.org/0000-0003-3627-7485 